\title{
EDITORIAL
}

\section{The dynamic yin-yang interaction of CXCR4 and CXCR7 in breast cancer metastasis}

\author{
Oriana E Hawkins and Ann Richmond* \\ See related research by Hernandez et al., http://breast-cancer-research.com/content/13/6/R128
}

\begin{abstract}
The contribution of CXCR7 to the tumor

microenvironment has introduced a new level of complexity to CXCL12 signaling in breast cancer. In the previous issue of Breast Cancer Research, Hernandez and colleagues delineate the roles of CXCR4 and CXCR7 in tumor invasion and metastasis. The authors demonstrate that co-expression of CXCR7 and CXCR4 results in inhibition of CXCL12-mediated invasion, reduced intravasation of tumor cells into the vasculature, and fewer lung metastases compared with parental tumors. The results of this study suggest the combination of small molecule inhibitors of CXCR4 and CXCR7 could dramatically reduce invasion, intravasation, and metastasis and could be highly beneficial for the treatment of invasive breast cancer.
\end{abstract}

The significance of CXCL12/CXCR4 signaling in breast cancer invasion and metastasis is widely appreciated. Recently, the contribution of the CXCL12 orphan receptor CXCR7 to the tumor environment has introduced a new level of complexity within the realm of chemokine/chemokine receptor signaling. Hernandez and colleagues have attempted to lift the veil and dissect the individual contributions of CXCR4 and CXCR7 to tumor invasion and metastasis. First, the authors demonstrate the potentiation of in vitro CXCL12/CXCR4mediated chemotaxis by the overexpression of CXCR7 in the MTLn3 JP cell line. The authors suggest this is due to the sequestration of CXCL12 by signaling-deficient CXCR7, which results in maintenance of a steep CXCL12 gradient [1]. These results are also consistent with reports that indicate CXCR7 as a sink for monomeric CXCL12, leaving dimeric CXCL12 to bind CXCR4 and mediate

*Correspondence: Ann.richmond@vanderbilt.edu

Department of Cancer Biology, Vanderbilt University School of Medicine, US Department of Veterans Affairs, 2220 Pierce Avenue, 771 Preston Research Building, Nashville, TN 37232-6840, USA signaling preferentially through the recruitment of $\beta$ arrestin, thus enhancing chemotaxis [2,3]. Although the authors do not address the formation of CXCR4/CXCR7 heterodimers by their transductants, it would seem to be a likely occurrence. Such heterodimers have been reported to constitutively recruit $\beta$-arrestin in response to CXCL12, potentiating chemotaxis when compared with CXCR4 homodimers or monomers [4,5].

Next, the authors demonstrate that while overexpression of CXCR4 in MTLn3 JP cells substantially increases invasion, co-expression of CXCR7 results in an inhibition of invasion in response to CXCL12 both in vitro and in vivo. In addition, CXCR7 overexpression reduced intravasation of tumor cells into the vasculature, which is probably due to the reduced ability of these cells to degrade the extracellular matrix. Following an evaluation of expression of matrix metalloproteinases, the authors identify MMP12 as upregulated by CXCL12 ligation of CXCR4 and diminished within the context of CXCR7 coexpression (Figure 1A and 1B) [1]. The authors cite a correlation between CXCR4 and MMP12 mRNA expression from the Oncomine database, but no such correlation with CXCR7 expression. These results are consistent with the observation that $\mathrm{G}_{\mathrm{i}}$-mediated signaling induced by monomeric CXCL12/CXCR4 could result in PKC $\delta$ mediated induction of MMP12 expression [6], whereas $\beta$-arrestin-mediated signaling is preferentially activated over canonical G-protein pathways when CXCR4/ CXCR7 heterodimers are expressed [5].

In agreement with reduced invasion, CXCR7-overexpressing tumors produced significantly fewer lung metastases than the parental MTLn3 JP tumors. The CXCR4overexpressing tumors produced fewer lung metastases than the parental tumor despite robust invasion and intravasation in vivo. The authors suggest anti-angiogenic properties of MMP12 could suppress seeding of the lung by CXCR4-overexpressing tumor cells [1]. An alternative explanation for this apparent paradox is offered by the research of Zabel and colleagues showing a requirement for CXCL12 binding to CXCR7 in CXCL12-mediated transendothelial migration in vitro (that is, extravasation; Figure $1 \mathrm{C}$ and 1D) $[7,8]$. 


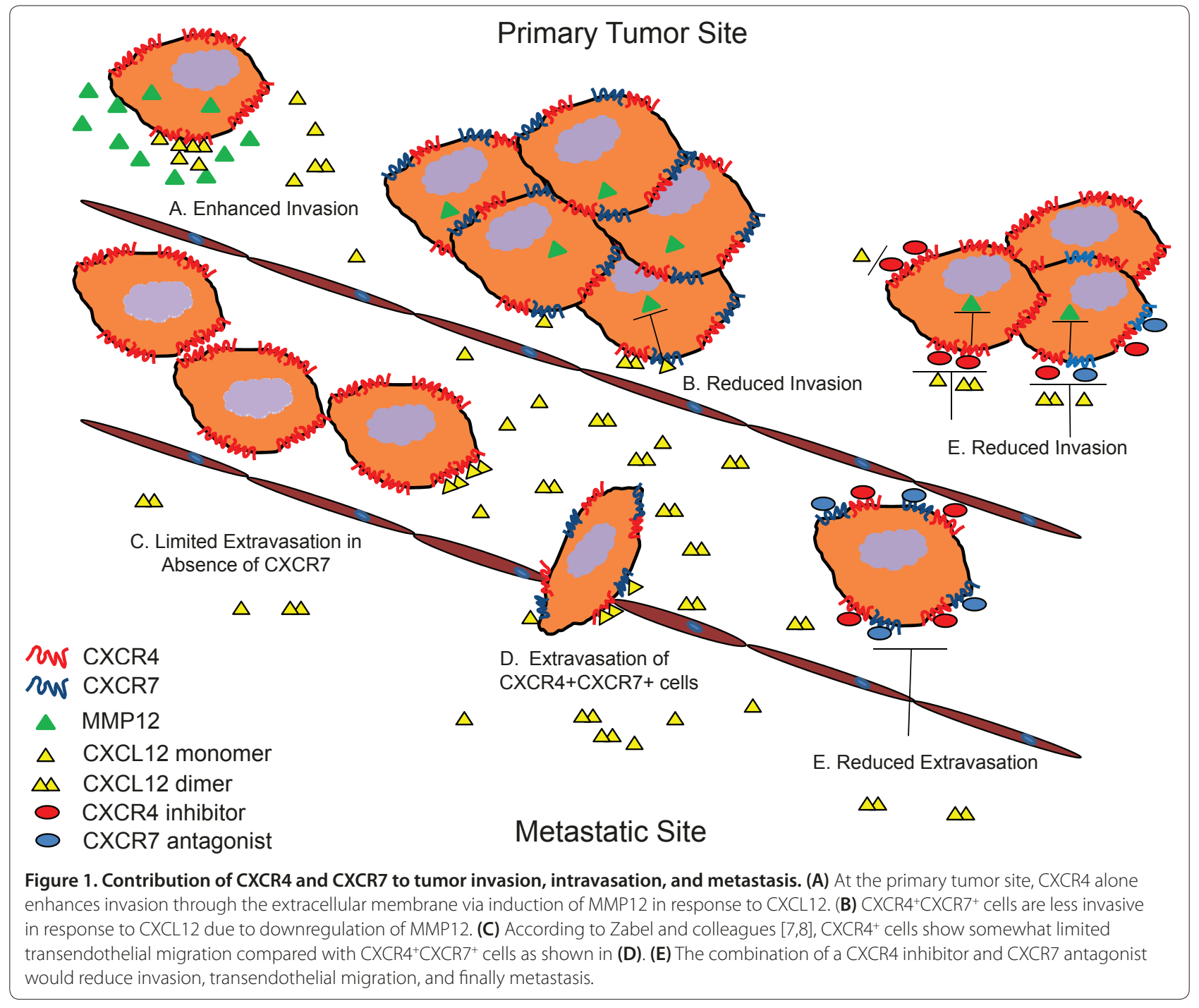

The results of the study by Hernandez and colleagues suggest a number of potential therapeutic modalities. The most obvious therapeutic targets currently in development are small molecule inhibitors of CXCR4 and CXCR7. Inhibition of CXCR4 using AMD3100 resulted in reduced chemotaxis and invasion in vitro and in vivo [1]. Antagonism of CXCR7 by compounds, such as CCX771, could reduce extravasation and thus metastasis [7]. Conceivably, the combination of such compounds could dramatically reduce invasion, intravasation, extravasation, and metastasis (Figure 1). Considering breast cancer metastasis is the leading cause of morbidity and mortality, such modalities could be highly beneficial for the treatment of invasive and preinvasive disease.

Competing interests

The authors declare that they have no competing interests.

Published: 26 January 2012
References

1. Hernandez L, Magalhaes MA, Coniglio SJ, Condeelis JS, Segall JE: Opposing roles of CXCR4 and CXCR7 in breast cancer metastasis. Breast Cancer Res 2011, 13:R128.

2. Ray P, Lewin SA, Mihalko LA, Lesher-Perez SC, Takayama S, Luker KE, Luker GD: Secreted CXCL12 (SDF-1) forms dimers under physiologic conditions. Biochem $J 2011$ [epub ahead of print].

3. Drury LJ, Ziarek JJ, Gravel S, Veldkamp CT, Takekoshi T, Hwang ST, Heveker N, Volkman BF, Dwinell MB: Monomeric and dimeric CXCL12 inhibit metastasis through distinct CXCR4 interactions and signaling pathways. Proc Natl Acad Sci U SA 2011, 108:17655-17660.

4. Decaillot FM, Kazmi MA, Lin Y, Ray-Saha S, Sakmar TP, Sachdev P: CXCR7/ CXCR4 heterodimer constitutively recruits $\beta$-arrestin to enhance cell migration. J Biol Chem 2011, 286:32188-32197.

5. Levoye A, Balabanian K, Baleux F, Bachelerie F, Lagane B: CXCR7 heterodimerizes with CXCR4 and regulates CXCL12-mediated G protein signaling. Blood 2009, 113:6085-6093.

6. Shukla A, Barrett TF, Nakayama KI, Nakayama K, Mossman BT, Lounsbury KM: Transcriptional up-regulation of MMP12 and MMP13 by asbestos occurs via a PKC $\delta$-dependent pathway in murine lung. Faseb J 2006, 20:997-999.

7. Zabel BA, Lewén S, Berahovich RD, Jaén JC, Schall TJ: The novel chemokine receptor CXCR7 regulates trans-endothelial migration of cancer cells. $\mathrm{Mol}$ Cancer 2011, 10:73. 
8. Zabel BA, Wang Y, Lewén S, Berahovich RD, Penfold ME, Zhang P, Powers J, Summers BC, Miao Z, Zhao B, Jalili A, Janowska-Wieczorek A, Jaen JC, Schall TJ: Elucidation of CXCR7-mediated signaling events and inhibition of CXCR4-mediated tumor cell transendothelial migration by CXCR7 ligands. J Immunol 2009, 183:3204-3211. doi:10.1186/bcr3092

Cite this article as: Hawkins OE, Richmond A: The dynamic yin-yang interaction of CXCR4 and CXCR7 in breast cancer metastasis. Breast Cancer Research 2012, 14:103. 